\title{
Correction to: Probability
}

\section{Correction to:}

Chapter 2 in: J. Unpingco, Python for Probability,

Statistics, and Machine Learning,

https://doi.org/10.1007/978-3-319-30717-6_2

The original version of the chapter was inadvertently published with incorrect integral equation. In Chapter 2, Page 55, the integral equation is updated. The chapter has now been corrected and approved by the author. 